Discrete Comput Geom 37:497-501 (2007)

DOI: $10.1007 / \mathrm{s} 00454-007-1346-3$

\title{
The Hadwiger Number of Jordan Regions Is Unbounded*
}

\author{
Otfried Cheong and Mira Lee \\ Division of Computer Science, \\ Korea Advanced Institute of Science and Technology, \\ Daejeon 305-701, South Korea \\ \{otfried,mira\}@tclab.kaist.ac.kr
}

\begin{abstract}
We show that for every $n>0$ there is a planar topological disk $A_{0}$ and $n$ translates $A_{1}, A_{2}, \ldots, A_{n}$ of $A_{0}$ such that the interiors of $A_{0}, \ldots, A_{n}$ are pairwise disjoint, but with each $A_{i}$ touching $A_{0}$ for $1 \leq i \leq n$.
\end{abstract}

\section{Introduction}

For any compact body $C \subset \mathbb{R}^{d}$, we define $H(C)$, the Hadwiger number of $C$, as the maximum number of mutually non-overlapping translates of $C$ that can be brought into contact with $C$ (see the survey by Zong [7]). Hadwiger [5] showed that for convex sets $C$ we have $H(C) \leq 3^{d}-1$ (using Minkowski's difference body method, see also [4]). The bound is tight for parallelepipeds [3], [4]. In the planar case it is known that $H(C)=6$ for every convex $C$ other than a parallelogram.

The arguments used in these results rely strongly on convexity. Considering the more general family of Jordan regions ${ }^{1}$ in the plane, Halberg et al. [6] could show that $H(C) \geq$ 6 holds for any Jordan region $C \subset \mathbb{R}^{2}$. More precisely, they showed that there exist six non-overlapping translates of $C$ all touching $C$ and whose union encloses $C$, where a set $A$ encloses a set $B$ if every unbounded connected set which intersects $B$ also intersects $A$. It seems therefore natural to conjecture that the Hadwiger numbers of Jordan regions in the plane are bounded by an absolute constant. Some more evidence for this conjecture is a result of Bezdek et al. [1] who showed that the maximum number of pairwise touching translates of a Jordan region $C$ in the plane is four. Since in this respect Jordan regions

\footnotetext{
* This research was supported by the Korea Research Foundation.

${ }^{1}$ A set $C \subset \mathbb{R}^{2}$ is a Jordan region or topological disk if it is bounded by a closed Jordan curve, or equivalently if it is homeomorphic to the unit disk.
} 
behave in the same way as convex sets, they ask the following question:

It seems reasonable to conjecture that $H(C) \leq 8$ for every planar Jordan region $C$. If this conjecture is false, is there an upper bound for $H(C)$ independent from the disk $C$ ?

[1, Problem 6.1]

As a first step in settling this conjecture, Bezdek could later show that $H(C) \leq 75$ if $C$ is a star-shaped Jordan region. The problem was picked up again by Brass et al. [2] (Problem 5 and Conjecture 6 in Section 2.4). We show here that the conjecture is not true in a strong sense: the Hadwiger number of Jordan regions in the plane is not bounded by any constant. For each $n>0$, we construct a Jordan region that admits $n$ mutually non-overlapping translates touching it.

The case of star-shaped Jordan regions remains open in the weaker sense of establishing the right constant: Brass et al. conjecture that this constant is 8 , but the best known upper bound is 75 .

\section{The Proof}

We consider the integer sequence $\mathcal{S}=s_{1}, s_{2}, \ldots$, where $s_{i}$ is the number of bits that must be counted from right to left to reach the first 1 in the binary representation of $i$ :

$$
\mathcal{S}=1,2,1,3,1,2,1,4,1,2,1,3,1,2,1,5,1,2,1,3,1,2,1,4,1,2,1,3,1,2,1,6, \ldots
$$

This sequence, which is also known as the ruler function, appears as sequence $A 001511$ in the on-line encyclopedia of integer sequences. ${ }^{2}$ We need the following property of this sequence.

Lemma 1. The prefix of length $k$ of $\mathcal{S}$ has the smallest sum among all subsequences of length $k$ of $\mathcal{S}$, for any $k>0$. Formally, for any $k, r>0$,

$$
\sum_{i=1}^{k} s_{i} \leq \sum_{i=r}^{r+k-1} s_{i}
$$

Proof. We proceed by induction. If $k=1$, the claim is true since $s_{1}=1 \leq s_{r}$. Assume now that $k>1$ and that the claim is true for all shorter prefixes. If $k$ is odd, then $s_{k}=1$, and by induction we have

$$
\sum_{i=1}^{k} s_{i}=1+\sum_{i=1}^{k-1} s_{i} \leq 1+\sum_{i=r}^{r+k-2} s_{i} \leq \sum_{i=r}^{r+k-1} s_{i} .
$$

It remains to consider even $k$. We observe that every odd term of $\mathcal{S}$ is equal to 1 , and that $\mathcal{S}$ has a nice recursive structure: removing all odd terms and subtracting 1 from all even

2 http: //www. research.att.com/ njas/sequences /A0 01511 . 
terms results in the same sequence $\mathcal{S}$ again. We therefore have

$\sum_{i=1}^{k} s_{i}=k / 2+\sum_{i=1}^{k / 2}\left(s_{i}+1\right)=k+\sum_{i=1}^{k / 2} s_{i} \leq k+\sum_{i=r^{\prime}}^{r^{\prime}+k / 2-1} s_{i}=k / 2+\sum_{i=r^{\prime}}^{r^{\prime}+k / 2-1}\left(s_{i}+1\right)=\sum_{i=r}^{r+k-1} s_{i}$,

where $r^{\prime}=\lceil r / 2\rceil$.

We can now describe our planar topological disk, or, more precisely, a two-parameter family of disks. For integers $m \geq 2$ and $n \geq 1$, the disk $D_{n}^{m}$ is the union of $2^{n}$ horizontal bars $B_{1}, \ldots, B_{2^{n}}$ and $2^{n}-1$ vertical connectors $V_{1}, \ldots, V_{2^{n}-1}$. All bars are axis-parallel rectangles of width $m$ and height 1 , all connectors are axis-parallel rectangles of width 1 . The height of connector $V_{i}$ is $s_{i}$ (the $i$ th term of our sequence $\mathcal{S}$ ). Informally, connector $V_{i}$ is placed above the rightmost unit square of bar $B_{i}$, while bar $B_{i+1}$ is placed to the right of the topmost unit square of connector $V_{i-1}$.

Formally, bar $B_{i}$ is the rectangle spanning the $x$-interval $[(i-1) m, i m]$ and the $y$ interval $\left[y_{i}, y_{i}+1\right]$, where $y_{i}=\sum_{j=1}^{i-1} s_{j}$. Connector $V_{i}$ spans the $x$-interval $[i m-1, i m]$ and the $y$-interval $\left[y_{i}+1, y_{i+1}+1\right]$.

Figure 1 shows $D_{n}^{m}$ for some values of $m$ and $n$.

We can give an alternative, recursive description of $D_{n}^{m}$, by observing that bars $B_{1}, \ldots, B_{2^{n-1}}$ and bars $B_{2^{n-1}+1}, \ldots, B_{2^{n}}$ of $D_{n}^{m}$ form two translates of $D_{n-1}^{m}$, connected by the single connector $V_{2^{n-1}}$. We can consider $D_{n}^{m}$ to consist of two translates of $D_{n-1}^{m}$, or four translates of $D_{n-2}^{m}$, or $2^{n-1}$ translates of $D_{1}^{m}$, or, in general, $2^{k}$ translates of $D_{n-k}^{m}$.

Lemma 2. Let $A$ and $A^{\prime}$ be translates of $D_{n}^{m}$, for $m, n \geq 2$, such that the first bar $B_{1}^{\prime}$ of $A^{\prime}$ is obtained from some bar $B_{r}$ of $A$ by a translation of $y^{*} \geq 1$ downwards and $1 \leq x^{*} \leq m-1$ to the right, where $1 \leq r \leq 2^{n}$. Then $A$ and $A^{\prime}$ have disjoint interiors.

Proof. Consider the vertical strip spanned by bar $B_{r-1+i}$ of $A$, for $1 \leq i \leq 2^{n}-r+1$. Since $1 \leq x^{*} \leq m-1$, this strip can intersect only bars $B_{i-1}^{\prime}$ and $B_{i}^{\prime}$ and connector $V_{i-1}^{\prime}$ of $A^{\prime}$. The highest $y$-coordinate in $B_{i-1}^{\prime} \cup V_{i-1}^{\prime} \cup B_{i}^{\prime}$ is $y_{i}+1$ with respect to the

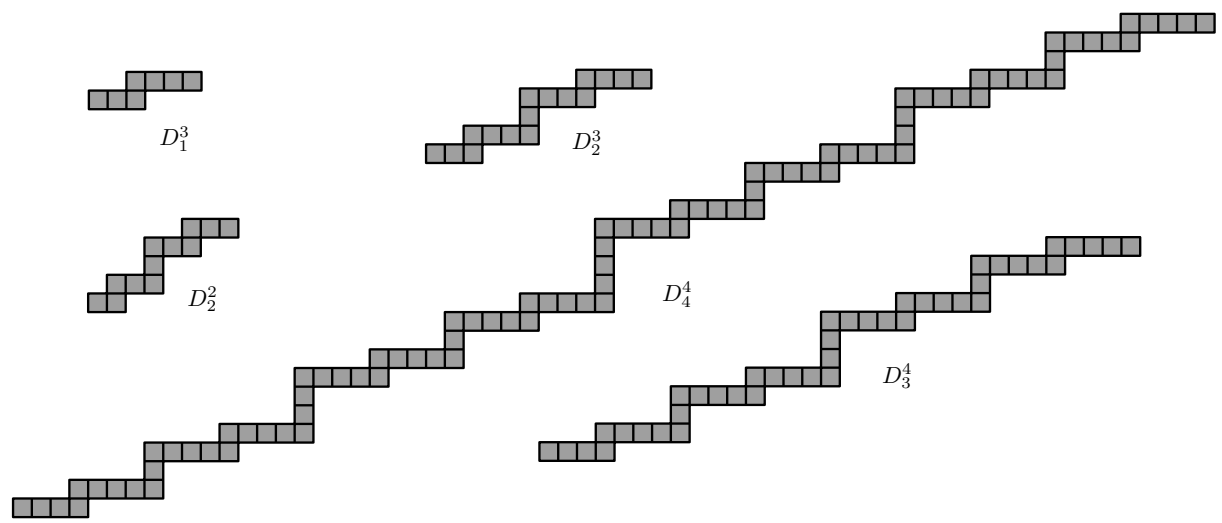

Fig. 1. Some $D_{n}^{m}$. 


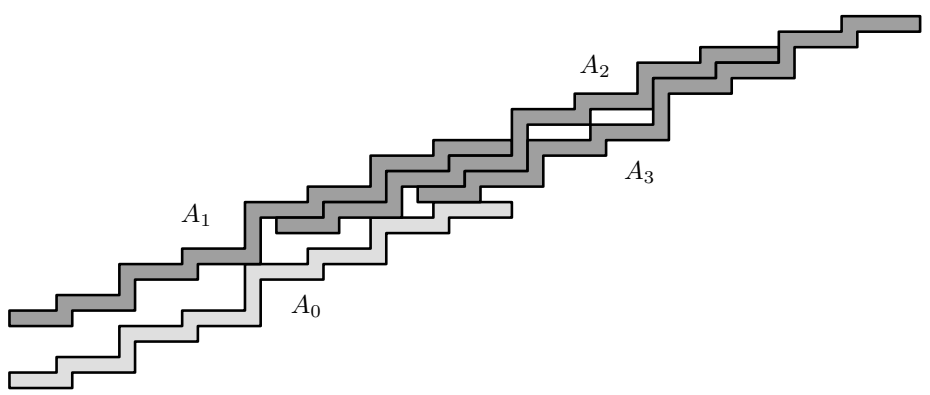

Fig. 2. The construction for $m=4, n=3$.

origin of $A^{\prime}$. By assumption, the origin of $A^{\prime}$ is at $y$-coordinate $y_{r}-y^{*} \leq y_{r}-1$, and so $B_{i-1}^{\prime} \cup V_{i-1}^{\prime} \cup B_{i}^{\prime}$ lies below the line $y=y_{r}+y_{i}$. On the other hand, the bottom edge of $B_{r-1+i}$ of $A$ has $y$-coordinate $y_{r-1+i}$. We now have

$$
y_{r-1+i}-\left(y_{r}+y_{i}\right)=\left(y_{r-1+i}-y_{r}\right)-y_{i}=\sum_{j=r}^{r+i-2} s_{j}-\sum_{j=1}^{i-1} s_{j} \geq 0
$$

by Lemma 1 . This implies that the interior of $B_{i-1}^{\prime} \cup V_{i-1}^{\prime} \cup B_{i}^{\prime}$ lies stricly below $B_{r-1+i}$, and the lemma follows.

We can now describe our construction of touching translates. We fix an integer $n>1$, and pick any integer $m \geq n$. Let $A_{1}$ be $D_{n}^{m}$. For $2 \leq i \leq n$ we obtain $A_{i}$ from $A_{i-1}$ as follows: The first (leftmost) copy of $D_{n+1-i}^{m}$ in $A_{i}$ is a translate of the second copy of $D_{n+1-i}^{m}$ in $A_{i-1}$, translated down by one and right by one.

We observe now that for any pair $1 \leq i<j \leq n$, the leftmost copy of $D_{n+1-j}^{m}$ in $A_{j}$ is a translate of some copy of $D_{n+1-j}^{m}$ in $A_{i}$, translated down by $j-i$ and right by $j-i$. Since $1 \leq j-i<n \leq m$, Lemma 2 implies that the interiors of $A_{i}$ and $A_{j}$ are disjoint.

Now let $A_{0}$ be a translate of $A_{1}$, translated downwards by $n+1$. See Fig. 2 for the entire construction for $m=4, n=3$.

It remains to show that $A_{i}$ touches $A_{0}$, but that their interiors are disjoint, for $1 \leq i \leq n$.

We pick some $i$. Let $D$ be the last (rightmost) copy of $D_{n+1-i}^{m}$ in $A_{0}$. Then the first copy $D^{\prime}$ of $D_{n+1-i}^{m}$ in $A_{i}$ can be obtained from $D$ by translating upwards by $n+1$, then downwards by $i-1$ and right by $i-1$. In other words, $D^{\prime}$ is obtained from $D$ by translating upwards by $n+2-i$ and right by $i-1$. Now the middle vertical segment of $D$ is a rectangle of height $n+2-i$, and so this translation brings $D$ and $D^{\prime}$ into contact. On the other hand, all other vertical segments in $D$ have length less than $n+2-i$, and so the interiors of $D$ and $D^{\prime}$ are disjoint. Finally, since $D$ is the rightmost part of $A_{0}$ and $D^{\prime}$ is the leftmost part of $A_{i}$, no other intersections between $A_{0}$ and $A_{i}$ are possible, and so their interiors are disjoint.

We summarize the result in the following theorem. Figure 3 shows a larger example.

Theorem 1. For any integer $n \geq 2$ and any integer $m \geq n$ there are $n+1$ translates $A_{0}, A_{1}, \ldots, A_{n}$ of $D_{n}^{m}$ whose interiors are pairwise disjoint, but such that $A_{0}$ touches every $A_{i}, 1 \leq i \leq n$. 


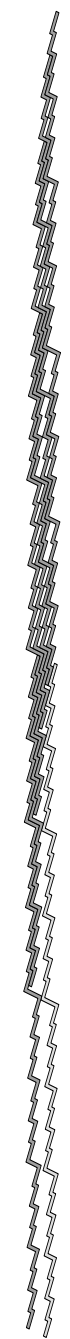

Fig. 3. The construction for $m=n=5$.

\section{References}

1. A. Bezdek, K. Kuperberg, and W. Kuperberg. Mutually contiguous translates of a plane disk. Duke Math. J., 78:19-31, 1995.

2. P. Brass, W. Moser, and J. Pach. Research Problems in Discrete Geometry. Springer-Verlag, New York, 2005.

3. H. Groemer. Abschätzungen für die Anzahl der konvexen Körper, die einen konvexen Körper berühren. Monatsh. Math., 65:74-81, 1961.

4. B. Grünbaum. On a conjecture of H. Hadwiger. Pacific J. Math, 11:215-219, 1961.

5. H. Hadwiger. Über Treffanzahlen be translationsgleichen Eikörpern. Arch. Math., 8:212-213, 1957.

6. C. J. A. Halberg, Jr., E. Levin, and E. G. Straus. On contiguous congruent sets in Euclidean space. Proc. Amer. Math. Soc., 10:335-344, 1959.

7. C. Zong. The kissing numbers of convex bodies - a brief survey. Bull. London Math. Soc., 30:1-10, 1998.

Received September 5, 2006, and in revised form February 15, 2007. Online publication April 26, 2007. 